\title{
Traslape de dieta entre el venado cola blanca y otros herbívoros en la Mixteca Poblana
}

\section{Dietary overlap among white tailed deer and other herbivores at la Mixteca Poblana}

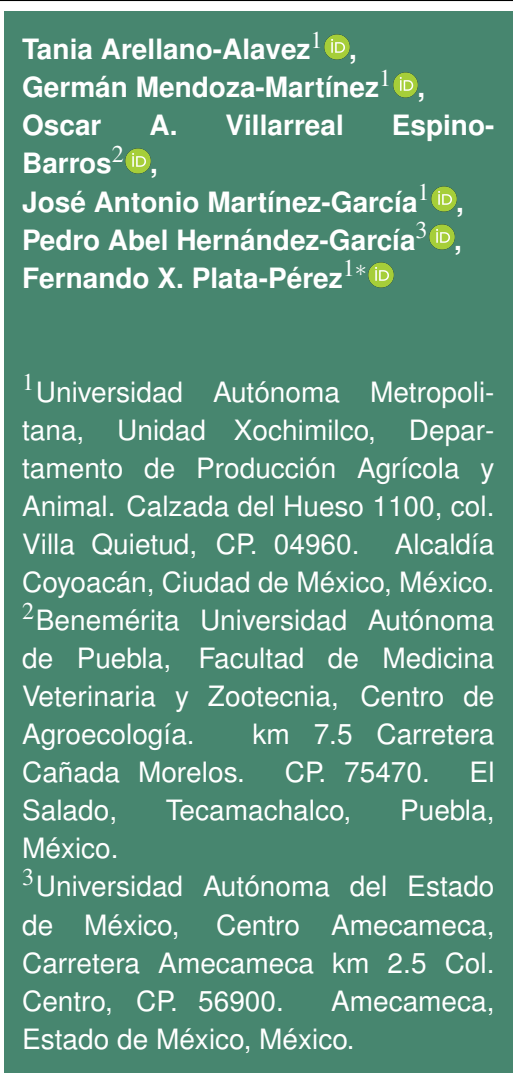

*Autor de correspondencia: ppfx2221@correo.xoc.uam.mx

Artículo científico

Recibido: 23 de julio 2021

Aceptado: 21 de septiembre 2021

Como citar: Arellano-Alavez T, Mendoza-Martínez G, Villarreal Espino-Barros OA, Martínez-García JA, Hernández-García PA, PlataPérez FX (2021) Traslape de dieta entre el venado cola blanca y otros herbívoros en la Mixteca Poblana. Ecosistemas y Recursos Agropecuarios 8(3): e3091. DOI: 10.19136/era.a8n3.3091
RESUMEN. El objetivo del estudio fue determinar la superposición de la dieta entre el venado cola blanca (Odocoileus virginianus) y otros herbívoros (lagomorfos, bovinos y equinos) caracterizando la dieta tanto de los cérvidos como la de los lagomorfos de la región. Se muestreó la vegetación y excretas encontradas en 15 transectos de $300 \mathrm{~m}$ de largo y $6 \mathrm{~m}$ de ancho. Mediante la técnica micro histológica se elaboró un catálogo de referencia vegetal y se analizaron las muestras fecales por especie para determinar la composición botánica de la dieta. De las 65 especies vegetales recolectadas presuntamente consumibles por los cérvidos, solo 16 fueron consumidas por estos. El análisis micro histológico reveló que la dieta de venados y lagomorfos coincide en una sola especie arbórea, un arbusto y una herbácea; pero, para los lagomorfos, el consumo de estos tres grupos vegetales representa más del $50 \%$ de la dieta; mientras que, para los caballos y bovinos, la dieta estuvo formada por una sola especie de herbáceas o gramíneas, respectivamente. El índice de Pianka entre el venado cola blanca y los lagomorfos fue de 0.26 , mientras que en comparación con el ganado bovino y equino fue de 0.04 en ambos casos. Como se esperaba del análisis micro histológico, la superposición de la dieta entre los ciervos y otras especies de herbívoros fue relativamente baja. Sin embargo, el traslape entre venado cola blanca y lagomorfos es del $26 \%$ y considerando el número estimado de individuos en la UMA puede ser un elemento de competencia importante.

Palabras clave: Densidad de población, Interacciones alimentarias, microhistología, ungulados, zonas áridas.

ABSTRACT. The objective of this study was to determine the dietary overlap between white-tailed deer (Odocoileus virginianus) and other herbivores (lagomorphs, cattle, and horses). Vegetation and excreta found in 15 transects $300 \mathrm{~m}$ long and $6 \mathrm{~m}$ wide were sampled. Using the micro histological technique, a plant reference catalog was elaborated and the fecal samples by species were analyzed to determine the botanical composition of the diet. It can be seen that, of 65 plant species collected presumably consumable by cervids, only 16 were consumed by them. The microhistological analysis revealed that the diet of deer and lagomorphs coincides in a single arboreal species, a shrub and a herbaceous one; However, in the diet of lagomorphs, the consumption of these three plant groups represents more than $50 \%$ of the diet, while, in the case of horses and cattle, the diet consisted mainly of a single species of herbaceous or grasses, respectively. The Pianka index between white-tailed deer and lagomorphs was 0.26 , while compared to cattle and horses it was 0.04 in both cases. As expected from microhistological analysis, the dietary overlap between deer and other herbivore species was relatively low. However, the overlap between white-tailed deer and lagomorphs is $26 \%$ and considering the estimated number of individuals in the UMA it may be an important element of competition.

Key words: Arid zones, density, dry season, feed interactions, micro histology, ungulate. 


\section{INTRODUCCIÓN}

En México, el aprovechamiento del venado cola blanca (Odocoileus virginianus) es regulado por el gobierno federal y se práctica en ranchos cinegéticos denominados "Unidades de Manejo para la conservación de la vida Silvestre"(UMAs). En la Mixteca Poblana, una de las regiones más pobres de México, en 1997 se inició el aprovechamiento en forma sustentable de una subespecie de venado cola blanca (Odocoileus virginianus mexicanus, Villarreal 2006). Las UMAs de esta región combinan la explotación extensiva de bovinos de carne y cabras con el aprovechamiento de venado cola blanca (Villarreal 1999, Villarreal et al. 2005), por esa razón los animales domésticos comparten su espacio y el alimento con la fauna silvestre en vida libre, por lo que dichos animales pueden representar un elemento de competencia por los recursos para el venado cola blanca (Faas y Weckerly 2010).

El venado cola blanca es un rumiante, considerado selector de concentrados, que consume plantas o partes de plantas fáciles de digerir, con alto contenido de proteínas y carbohidratos solubles intracelulares (Pruszenski y Hernández 2020). Normalmente ingiere una cantidad muy reducida de gramíneas (Plata et al. 2009). En contraste, el ganado doméstico (bovinos, ovinos y equinos) se alimentan preferentemente de pastos y se les considera como consumidores de forraje (Rivero et al. 2021); sin embargo, en zonas áridas, las herbáceas y arbustos pueden representar desde un 5 hasta un 15\% de su dieta (Clark et al. 2013).

En el desierto de Sonora (Condado de Imperial, California, EE. UU.) se han realizado algunos estudios sobre la superposición dietética entre el venado bura (Odocoileus hemionus) y los burros salvajes (Equus asinus), los resultados de este estudio sugieren que los équidos a pesar de ser consumidores de forraje con poca preferencia por los arbustos pueden consumir una proporción significativa del alimento de los cérvidos (Marshal et al. 2012). Por otro lado, los sitios con alta productividad vegetal se asocian con una gran cantidad de especies de pequeños mamíferos, principalmente lagomorfos (Mc
Cain et al. 2018). Algunos autores mencionan que algunas especies de estos animales son consumidores generalistas y que se encuentran en bosques de sucesión temprana (Crawford et al. 2018). Sin embargo, existen otras especies de lagomorfos que consumen principalmente herbáceas, arbustivas y plantas leñosas (Kontsiotis et al. 2015) dependiendo de la disponibilidad de especies vegetales en algunas épocas del año pueden consumir una gran cantidad de arbustos (40\%) en su dieta (Howard et al. 1987, Kontsiotis et al. 2015). Como consecuencia se convierten en invasores que ocasionan la degradación de la vegetación y la extinción de especies vegetales, con efectos en cascada en mamíferos locales, aves y reptiles (Williams et al. 1995). Adicionalmente, compiten con el ganado doméstico y destruyen cultivos, lo cual tiene un impacto negativo significativo sobre la economía local (Kontsiotis et al. 2015). En el estado de Puebla se han identificado siete especies de lagomorfos (Romerolagus diazi, Lepus callotis, $L$. californicus, Sylvilagus audubonii, S. brasiliensis, S. cunicularius y el $S$. floridanus orizabae; CONABIO 2011). Sin embargo, en las regiones áridas del estado no se han caracterizado sus dietas. Considerando que tanto el ganado doméstico como los lagomorfos pueden competir ya sea por el alimento o por el espacio y pueden reducir la capacidad de carga para el venado cola blanca. El objetivo del estudio fue determinar la superposición de la dieta entre el venado cola blanca (Odocoileus virginianus) y otros herbívoros (lagomorfos, bovinos y equinos) caracterizando la dieta tanto de los cérvidos como la de los lagomorfos de la región.

\section{MATERIALES Y MÉTODOS}

\section{Área de estudio}

El estudio se realizó en la UMA "Aminitlán"perteneciente al ejido San Miguel, localizado en el Municipio de Chiautla de Tapia en la Mixteca Poblana (Figura 1). Chiautla de Tapia se ubica entre los paralelos $18^{\circ} 06^{\prime}$ y $18^{\circ} 28^{\prime}$ de latitud norte, y los meridianos $98^{\circ} 23^{\prime}$ y $98^{\circ} 49^{\prime}$ de longitud oeste, la altura sobre el nivel del mar va de 800-2 000 msnm. El clima es cálido subhúmedo 


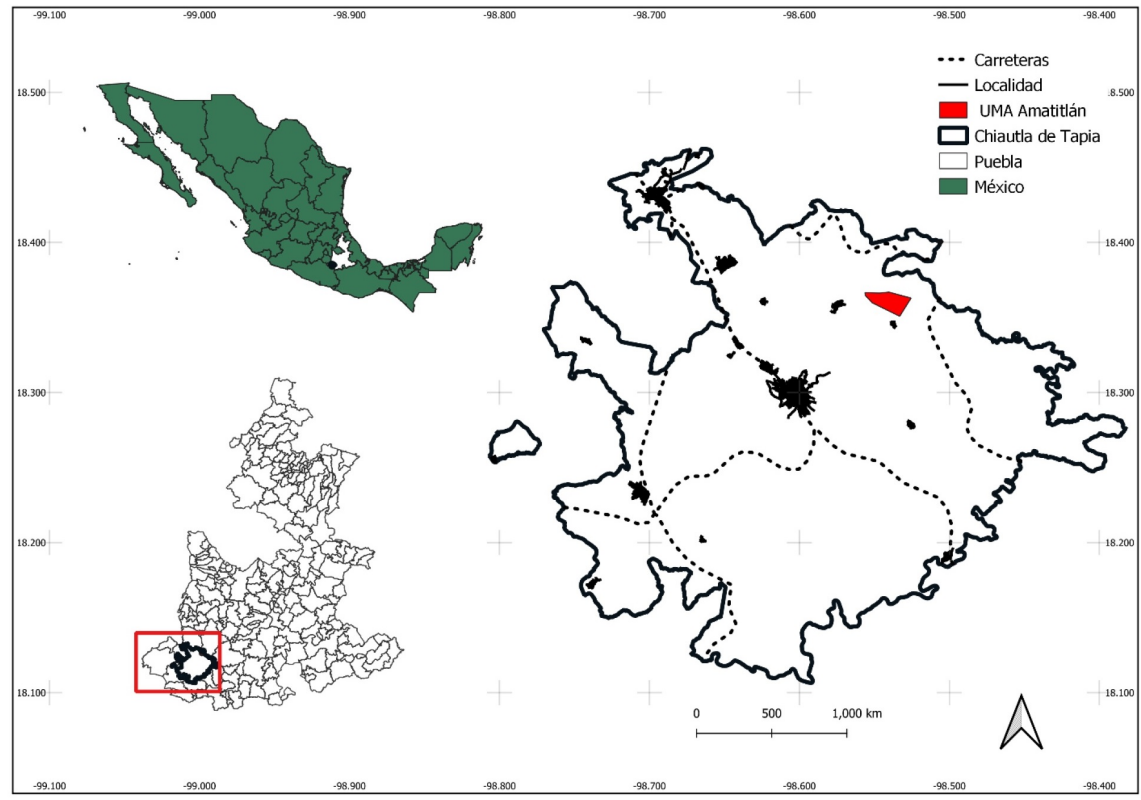

Figura 1. UMA Aminitlán, en el Ejido San Miguel, Municipio de Chiautla de Tapia en la Mixteca Poblana, Puebla, México.

con lluvias en verano y semiárido y caluroso en la estación seca, con temperatura anual entre 18 y 26 ${ }^{\circ} \mathrm{C}$. La precipitación anual varía entre 700 y $900 \mathrm{~mm}$ con una época de seca bien caracterizada y una época lluviosa en el verano (INEGI 2009). Los principales tipos de vegetación son el bosque caducifolio y el bosque caducifolio tropical (Guízar-Nolazco et al. 2010, Villarreal et al. 2011). Esta UMA es extensiva, tiene un área total de 1250 ha con 771 ha en manejo comunal y su finalidad principal es el aprovechamiento extractivo del venado cola.

\section{Muestreos de vegetación}

Con la finalidad de identificar las especies vegetales consumidas por las diferentes especies animales presentes en el área, se establecieron 15 transectos de $300 \mathrm{~m}$ de longitud por seis metros de ancho. Usando mapas de la UMA y siguiendo las recomendaciones de Foster et al. (2019), se trazó el polígono de la superficie que conforma la Unidad. La asignación del punto de inicio de cada vector fue completamente al azar, considerando que su distribución cubriera la totalidad de la UMA.

\section{Análisis de la diversidad de la vegetación}

Utilizando como referencia los puntos de inicio de transecto y siguiendo la dirección de estos, a cada 100 metros se marcaron cuadros de $4 \mathrm{~m} \times 4 \mathrm{~m}$ para tener una superficie de $16 \mathrm{~m}^{2}$ (Bullock 1999). Dentro de estos sitios se colectaron muestras de las especies vegetales. El muestreo se realizó por estrato vegetativo (pastos, herbáceas, arbustivas y arbóreas). Para caracterizar las especies de porte erecto (árboles y arbustos) se recolectaron muestras del material vegetal consumible por los venados (hojas, flores, bellotas o frutos) que se encontraban por debajo de $1.5 \mathrm{~m}$ de altura (Plata et al. 2011). Con los valores obtenidos a partir de esta información se estimó el número de plantas por hectárea, la riqueza y el índice de Shannon (Buckland et al. 2005, Elzinga et al. 2001, Krebs 2006).

\section{Estimación del número de animales}

Con base en lo establecido por Marques et al. (2001) los transectos establecidos se fragmentaron utilizando 10 segmentos por transecto de 300 $\mathrm{m}$. En dichos segmentos se geoposicionaron los grupos fecales de las diversas especies animales que aparecieron después de la limpieza de estos. La den- 
sidad de población de venado cola blanca se estimó utilizando el método de pellets (Mayle et al. 1999) y el cálculo de la densidad de población se realizó con la metodología de Mandujano (2014). Para tener control sobre la deposición de excretas (tiempo y número de muestras), previo a la colecta se limpiaron completamente los transectos, posteriormente se colectaron todas las excretas acumuladas a lo largo de 60 días. Para venados se consideró un grupo fecal cuando estaba formado por al menos 15 gránulos juntos con tamaño y forma similar (Sánchez-Rojas et al. 2004). El modelo de Mandujano (2014) requiere tres tasas diferentes de defecación (12.2, 19.3 y 26.4) para después arrojar el número de animales promedio en base a las tres estimaciones previas. La estimación de la densidad de los lagomorfos se realizó con el modelo de Mutze et al. (2014), considerando que un grupo fecal cuando estaba formado por al menos 40 gránulos juntos con tamaño y forma similar. La estimación de la densidad de equinos se realizó utilizando el modelo de Zabek et al. (2016), considerando una tasa de defecación de 7.9 grupos de excretas por día. La estimación de la densidad de los bovinos se realizó con una tasa de defecación de 11.9 grupos de excretas por día (Julander 1955).

\section{Catálogo de referencia}

Una de las estrategias más adecuadas para estudiar la dieta de animales en vida libre, es el análisis microscópico de las heces de los animales de las especies estudiadas, dicho estudio debe considerar los cambios en la abundancia de las diferentes especies vegetales en las diferentes épocas del año y el conocimiento de los cambios en sus características morfológicas después de la degradación por el proceso digestivo que sufren después de ser ingeridas. Para ello, se elaboró un catálogo de referencia de plantas (Johnson et al. 1983). Se recolectaron muestras de todas las especies vegetales presentes en los cuadros de muestreo y se realizó su identificación taxonómica en los herbarios de la Benemérita Universidad Autónoma de Puebla y en la Escuela Nacional de Ciencias Biológicas (ENCB). Previo al envío, las muestras fueron etiquetadas de manera individual incluyendo las coordenadas de referencia y altitud del lugar en que se colectaron. Se implementó la técnica microhistológica (Holechek, 1982), para lo cual, las muestras se desecaron siguiendo la metodología de Peña y Habib (1980), para su posterior molido, tamizado y preparado en ebullición con solución de hidróxido de sodio $(\mathrm{NaOH})$ e hipoclorito de sodio ( $\mathrm{NaClO}$ ). Se elaboraron las laminillas de referencia por duplicado de cada muestra tratada, etiquetándose con su respectivo nombre y clave.

Las laminillas de excretas se deshidrataron con la misma técnica (Peña y Habib 1980) y antes del molido y tamizado se separaron las semillas presentes en las excretas para su posterior identificación. Se sometieron al mismo lavado y ebullición que la efectuada en los vegetales, así como la elaboración de laminillas de cada especie animal. La revisión microscópica de las laminillas de las muestras vegetales y animales se realizó en el laboratorio de Citoquímica de la ENCB, utilizando el software PIXEL PRO ${ }^{\circledR}$ de Labomed.

\section{Traslape de dietas}

Con la finalidad de identificar las diferencias entre las dietas de las diferentes especies herbívoras que se encuentran en la UMA estudiada, se realizó un análisis micro-histológico (Johnson et al. 1983) de las excretas de caballos, bovinos, lagomorfos y venados presentes dentro del área de muestreo de cada uno de los transectos. Tanto las muestras de heces como de material vegetativo fueron caracterizadas siguiendo la metodología recomendada por Holechek (1982). El análisis microscópico del material se realizó en el laboratorio de Citoquímica de la ENCB y las microfotografías fueron realizadas utilizando el software PIXEL PRO ${ }^{\circledR}$ de Labomed. La superposición de la dieta entre el venado cola blanca y los otros herbívoros se calculó utilizando el índice de Pianka (Martínez-García et al. 2012) de acuerdo con la siguiente ecuación:

$$
0_{j k}=\frac{\sum n P_{i j} P_{i k}}{\sqrt{\sum P_{i i}^{2}} \sum P_{i k}^{2}}
$$

Donde: 0 es el grado de traslape de la dieta o el índice de Pianka, $\mathrm{P}_{i i}$ y $\mathrm{P}_{i k}$ representan las proporciones de alimento i que se encuentra en la dieta 
de las especies j y k, respectivamente. El índice de Pianka varía entre 0 (sin superposición) y 1 (superposición total).

\section{RESULTADOS}

\section{Análisis de la diversidad de la vegetación}

El índice de Shannon fue de 3.28 y el índice de Margalef de 10.54 mientras que el listado de especies vegetales encontradas en la UMA (Tabla 1), muestra la presencia de ocho familias y 65 especies.

\section{Estimación del número de individuos}

Se encontraron 111 grupos de excretas de venado, 22 de lagomorfos, tres de bovinos, una de caballos y seis de otras especies (cuatro de zorro, Lycalopex culpaeus; una de coyote, Canis latrans; una de roedor ND). Con esta información y considerando el tiempo en el que se recolectaron luego de la limpieza del área (60 días) se estimó el número total individuos. Para venados fue de 4.96 ( \pm 3.8 ), lagomorfos 132.27 ( \pm 126.4 ); vacunos, tres y equinos uno ind $/ \mathrm{km}^{2}$. En estas últimas especies (vacunos $\mathrm{y}$ equinos) debido al tamaño de la muestra la varianza fue cero.

\section{Características de la dieta de las especies evaluadas}

En la composición de la dieta del venado cola blanca en la zona, así como la de los lagomorfos, ganado vacuno y caballos domésticos, se puede observar que, aunque la dieta de los venados y los lagomorfos coinciden en solo una especie de arbóreas, una de arbustivas y una de herbáceas en la dieta de los lagomorfos el consumo de estos tres grupos vegetales representa más del $50 \%$ de la dieta, mientras que en el caso de los equinos y los bovinos la dieta fue formada fundamentalmente por una sola especie de herbácea o de pasto respectivamente (Tabla 2). Con la información sobre la dieta se calculó el índice de Pianka (superposición) para la dieta de ciervos con otros herbívoros, como se esperaba del análisis micro-histológico, el traslape de dieta entre venados y los herbívoros domésticos fue relativamen- te bajo (Tabla 3); sin embargo, con los lagomorfos el traslape fue del $26 \%$ que resulta en una superposición importante de las dietas entre ambas especies.

\section{DISCUSIÓN}

\section{Análisis de la diversidad de vegetación}

El índice de Shannon reportado en este trabajo es ligeramente más bajo al reportado por CamposSalas et al. (2016), quienes reportaron un índice de Shannon de 3.36 en la región Tehuacán, Puebla. Al respecto se sabe que este índice está asociado a una baja perturbación del suelo debido al manejo que la comunidad hace para preservar las plantas (Casas et al. 2006) y al hecho de que al ser una UMA para la extracción de venado cola blanca la cantidad de actividades que perturban (Siembra y cultivo) son reducidas. El índice de Margalef obtenido es un valor que refleja una riqueza muy alta (65 especies) y la baja perturbación del área. Estas especies pertenecen en su mayoría a familias vegetales que coinciden con las familias reportadas por Guízar-Nolazco et al. (2010) quienes para el sur del estado de Puebla reportan 360 especies que representaban a 225 géneros pertenecientes a 77 familias. Es importante señalar que; los cambios en la distribución de las especies dentro de una comunidad vegetal están asociados a la fertilidad del suelo, la densidad de plantas y el impacto de los depredadores (herbívoros), los cuales reducen o aumentan la cantidad de nutrientes disponibles para las plantas (Aarssen et al. 2003). De acuerdo con Matteucci et al. (1999), la vegetación está formada por un patrón complejo de tipos fisonómicos que son el resultado de la heterogeneidad ambiental, la cual refleja los efectos del clima, la orientación, el relieve, el tipo de suelo y el agua disponible, la cual incluye la precipitación, la captación por las raíces y la evapotranspiración. También, dicho patrón puede ser modificado por las relaciones de sinergia y competencia que existen entre las especies vegetales en un sitio (Austin 1999, Huerta-Martínez et al. 2004, Otto et al. 2001). 
Tabla 1. Relación de especies vegetales encontradas en la UMA (65 especies).

\begin{tabular}{|c|c|c|c|c|c|}
\hline Nombre científico & Familia & Nombre común & & Número de individuos por: & \\
\hline Nombre científico & Familia & Nombre común & $\begin{array}{r}\text { Densidad, } \\
\text { Individuos/ } \mathrm{Ha}\end{array}$ & $\begin{array}{r}\text { Abundancia Densidad, } \\
\text { relativa, \% }\end{array}$ & Individuos/ Km \\
\hline Serjania schiedeana & Sapindaceae & Temacate de 3 costillas & 187.5 & 5.13 & 18750 \\
\hline Pterium spp & & Helecho & 20.875 & 0.57 & 2087.5 \\
\hline Chlorophora tinctoria $L$. & Moraceae & Mora & 10.375 & 0.28 & 1037.5 \\
\hline Lysiloma divaricatum & Fabaceae & Tepehuaje & 52.125 & 1.43 & 5212.5 \\
\hline Eysenhardtia polystachya & Fabaceae & Palo dulce & 93.75 & 2.56 & 9375 \\
\hline Lysiloma divaricatum & Fabaceae & Tlahuitole & 104.125 & 2.85 & 10412.5 \\
\hline Thevetia ovata & Apocynaceae & Yoyote & 52.125 & 1.43 & 5212.5 \\
\hline Malvella leprosae & Malvaceae & Hierba de oreja & 10.375 & 0.28 & 1037.5 \\
\hline Serjania schiedeana & Sapindaceae & Temecate de cenizo & 41.625 & 1.14 & 4162.5 \\
\hline Montanoa speciosa & & Aclina & 312.5 & 8.55 & 31250 \\
\hline Ipomoea murucoides & & Cazahuate & 229.125 & 6.27 & 22912.5 \\
\hline Euphorbia schlechtendalii & & Estomeca & 260.375 & 7.12 & 26037.5 \\
\hline Whalteria americana & & Jehuite & 10.375 & 0.28 & 1037.5 \\
\hline Crotalaria acapulcensis & & Confetillo & 52.125 & 1.43 & 5212.5 \\
\hline Melampodium sp. & & Rosa amarilla & 135.375 & 3.70 & 13537.5 \\
\hline Serjania racemosa schum & & Temecate de bejuco & 10.375 & 0.28 & 1037.5 \\
\hline Heliocarpus terebinthinaceus & Compositae & Cuahualahua & 10.375 & 0.28 & 1037.5 \\
\hline Acacia farnesiana & & Huizache & 10.375 & 0.28 & 1037.5 \\
\hline Fouquieria formosa & & Vara de ocote & 104.125 & 2.85 & 10412.5 \\
\hline Acacia subangulata & & Sierrecilla & 145.875 & 3.99 & 14587.5 \\
\hline Malpigia mexicana & Malphigiaceae Huajocote & 10.375 & 0.28 & 1037.5 & \\
\hline Peperomia donaguiana & & Aclapanchi & 10.375 & 0.28 & 1037.5 \\
\hline Ceiba parvifolia & & Pochote & 41.625 & 1.14 & 4162.5 \\
\hline Leucaena leucocephala & & Huaje de caballo & 10.375 & 0.28 & 1037.5 \\
\hline Verbesiana crocata & Asteraceae & Capitonejo & 20.875 & 0.57 & 2087.5 \\
\hline Mimosa sp. & & Uña de gato & 62.5 & 1.71 & 6250 \\
\hline Trichilia hirta & Meliaceae & Tapa queso & 10.375 & 0.28 & 1037.5 \\
\hline Pinus patula & & Ocote & 10.375 & 0.28 & 1037.5 \\
\hline Tournefortia hirsutissima L & & Tlachichinole & 10.375 & 0.28 & 1037.5 \\
\hline Equisetum hyemale & & Carrizillo & 41.625 & 1.14 & 4162.5 \\
\hline Serjania racemosa schum & Sapindaceae & Hierba de golpe & 20.875 & 0.57 & 2087.5 \\
\hline Melinis repens & & Zacate criollo & 41.625 & 1.14 & 4162.5 \\
\hline Bunchosia lanceolada & & Nanche de zorra & 20.875 & 0.57 & 2087.5 \\
\hline Cordia curassavica & & Vara negra & 10.375 & 0.28 & 1037.5 \\
\hline Spondeas purpurea & & Cirguelillo & 10.375 & 0.28 & 1037.5 \\
\hline Aristida adscencionis & & Zacate grueso & 10.375 & 0.28 & 1037.5 \\
\hline Baccharis Heterophylla & & Escobilla & 10.375 & 0.28 & 1037.5 \\
\hline Selaginella lepidophylla & & Huaje de leon & 10.375 & 0.28 & 1037.5 \\
\hline Tecoma stans (L.) & & Istoncle & 10.375 & 0.28 & 1037.5 \\
\hline Solanum laxum Spreng. & & Enredadera & 20.875 & 0.57 & 2087.5 \\
\hline Acacia spp & & Cubata & 20.875 & 0.57 & 2087.5 \\
\hline Melothria pendula & & Pepino de cerro & 10.375 & 0.28 & 1037.5 \\
\hline Calea zacatechichi & & Zacatechichin & 52.125 & 1.43 & 5212.5 \\
\hline Oenothera pubescens & Onagraceae & Sin vergüenza & 20.875 & 0.57 & 2087.5 \\
\hline Selaginella lepidophylla & Selaginaláceas & Doradilla & 20.875 & 0.57 & 2087.5 \\
\hline Asparagaceae & & Vara de cura & 41.625 & 1.14 & 4162.5 \\
\hline Paspalum plicatum & & Pajon & 20.875 & 0.57 & 2087.5 \\
\hline Amaranthus spp & & Huaquelite & 10.375 & 0.28 & 1037.5 \\
\hline Eysenhardtia polystachya & & Palo de cera & 20.875 & 0.57 & 2087.5 \\
\hline Physalis $L$. & & Tomatón & 10.375 & 0.28 & 1037.5 \\
\hline Psidium guajava & & Manzana & 10.375 & 0.28 & 1037.5 \\
\hline Lippia graveolens & Verbenaceae & Oreganillo & 10.375 & 0.28 & 1037.5 \\
\hline Cynodon dactylon & & Zacate de gramilla & 10.375 & 0.28 & 1037.5 \\
\hline Leucaena sp. & & Huaje & 10.375 & 0.28 & 1037.5 \\
\hline Acacia farnesiana & & Espinillo & 10.375 & 0.28 & 1037.5 \\
\hline Serjania brachycarpa & & Temecate morado & 10.375 & 0.28 & 1037.5 \\
\hline Houttuynia cordata & & Hierba de pescado & 10.375 & 0.28 & 1037.5 \\
\hline \multirow[t]{6}{*}{ Nicotiana glauca } & & Tabacon & 10.375 & 0.28 & 1037.5 \\
\hline & & Calpicerán & 10.375 & 0.28 & 1037.5 \\
\hline & & Hierba & 697.875 & 19.09 & 69787.5 \\
\hline & & Hierbas & 10.375 & 0.28 & 1037.5 \\
\hline & & Tapapitallo & 72.875 & 1.99 & 7287.5 \\
\hline & & No identificado & 281.25 & 7.69 & 28125 \\
\hline
\end{tabular}

\section{Estimación del número de individuos}

La densidad poblacional expresada por $\mathrm{km}^{2}$ es de 4.96 venados, la cual se encuentra dentro del rango superior reportado para venado cola blanca en la Mixteca (Puebla) y que va, de 2.2 a 7.8 venados $/ \mathrm{km}^{2}$ (Villarreal et al. 2011). Esta densidad sugiere que es posible aumentar el aprovechamiento del venado cola blanca dentro de la UMA mejorando el plan de manejo, considerando que para asegurar la viabilidad y supervivencia demográfica y genética de la población con un $95 \%$ de confianza a 100 años, el tamaño poblacional mínimo viable va de 200 a 500 individuos, y que el mínimo de individuos para garantizar la supervivencia de esta especie es de 1.33 animales por $\mathrm{km}^{2}$ (Mandujano 2011). Adicionalmente, el alto número de individuos de venado cola- 
Tabla 2. Composición botánica de la dieta (\%), del venado cola blanca, lagomorfos, equinos y bovinos en una UMA de la Mixteca Poblana.

\begin{tabular}{lllcccc}
\hline Nombre científico & Nombre común & Tipo vegetal & Venado & Lagomorfo & Equino & Bovino \\
\hline Acacia pennulata & Cubata & Arb & 1.96 & 14.29 & - & - \\
Andropogon glomeratus & Zacate Bramilia & Gram & 1.96 & 28.57 & - & 100 \\
Calea zacatechichi & Zacate chichi & Gram & 3.92 & 14.29 & - & - \\
Ceiba parvifolia & Pochote & Arb & 31.37 & - & - & - \\
Euphorbia schlechtendalii & Tomeca & arbu & 9.80 & 28.57 & & \\
Eysenhardtia polystachya & Palo dulce & Arb leg & 9.80 & - & - & - \\
Crotalaria acapulcensis & Confetillo & Herb leg & 3.92 & - & - & - \\
Ipomea murucoides & Cazahuate & arb & 1.96 & - & - & - \\
Melampodium & Rosa Amarilla & arbu & 11.76 & - & - & - \\
Montanoa speciosa & Acahual & herb & 5.88 & - & - & - \\
Peperomia donaguina & Quelitillo de venado & herb & 3.92 & - & - & - \\
Calystegia sepium & Carrizillo & herb & 3.92 & - & - & - \\
Selaginella lepidophylla & Doradilla & herb & 3.92 & 14.29 & - & - \\
Serjania racemosa schum & Hierba de golpe & herb & 1.96 & - & 100.00 & - \\
Spondeas pupurea & Ciruelo & arb & 1.96 & - & - & - \\
Chlorophora tinctoria L. & Mora & arb & 1.96 & - & - & - \\
\hline Arb Arbórea; Gram $=$ gramínea; Arbu = Arbustiva; leg = leguminosa; Herb = herbácea & &
\end{tabular}

Tabla 3. Índice de Pianka entre la dieta de venado cola blanca y otros herbívoros.

\begin{tabular}{lc}
\hline Venado cola blanca y & Índice \\
\hline Lagomorfos & 0.26 \\
Equinos & 0.04 \\
Bovinos & 0.04 \\
\hline
\end{tabular}

blanca muestra que los cambios en la diversidad y abundancia vegetal que resultan de la perturbación antropogénica de la UMA son bajos, y están asociados a la presencia del venado (Burnett et al. 2020). El número de lagomorfos reportados en este trabajo es mayor al reportado por Portales et al. (2016), pero se debe a que esos autores reportaron exclusivamente la densidad de la liebre de cola-negra (Lepus californicus) en el desierto de Chihuahua y no reportaron el total de lagomorfos encontrados en dicha área, por el contrario, Martínez-Calderas et al. (2016) reportaron un número muy similar de indicios fecales a los encontrados en este trabajo, lo cual sugiere que la diferencia en el tamaño poblacional está asociada al método de estimación y al número de especies evaluadas.

En la composición de la dieta del venado cola-blanca, la planta que tuvo mayor frecuencia en las heces fue la Ceiba parvifolia (arbórea, 31.37\%), seguida de Melampodium (arbustiva, 11.76\%), la Euphorbia schlechtendalii (arbustiva, 9.8\%) y la Eysenhardtia polystachya (arbórea leguminosa, 9.8\%).
Mientras que, en los lagomorfos, la plantas con mayor porcentaje de aparición fueron la Euphorbia schlechtendalii (arbustiva, 28.57\%) y el Andropogon glomeratus (gramínea, 28.57\%), juntos representan el $57.14 \%$ de la dieta. Por otro lado, los equinos y los bovinos consumen Serjania racemosa (herbácea, 100\%) y el Andropogon glomeratus (gramínea, 100\%) respectivamente, ambas especies son parte tanto de la dieta de los venados como la de los lagomorfos. Al respecto, Kingery et al. (1996) al evaluar la posibilidad de que la dieta del bovino, el alce (Cervus elaphus nelsoni) y el venado cola blanca se traslaparan y compitieran entre ellos reduciendo encontraron que la dieta del bovino y el alce se traslapan hasta en un $88 \%$, dependiendo de la época del año. Pero cuando compararon al bovino con el venado cola blanca sus resultados mostraron que dichas especies se llegan a traslapar en porcentajes menores al $21 \%$, lo que incrementa la capacidad de carga. También encontraron que existe una interacción significativa entre la comunidad vegetal y el tipo de dieta que selecciona una especie determinada. Esta selectividad está vinculada al peso metabólico de la especie, debido a que animales con un menor tamaño y un mayor gasto energético están obligados a consumir una dieta con una mayor concentración de nutrientes para cubrir sus requerimientos metabólicos de mantenimiento. En México, trabajos realizados por Martínez et al. 
(1997) muestran que, en los venados, la dieta cambia de acuerdo con el sistema de pastoreo existente en la zona que habita. Sin embargo, en los sistemas evaluados la cantidad de arbustivas consumida rebasa al $50 \%$ del total de la dieta consumida.

Al comparar la superposición de la dieta entre conejos (Lepus timidus) y corzo (Capreolus capreolus), los conejos consumieron una dieta con el 55\% de arbustivas cuando estaban en presencia de este cérvido, mientras que el corzo consumió una dieta con un $40 \%$ de arbustivas y el $60 \%$ de gramíneas (Hulbert y Andersen 2001). En otro trabajo realizado en el desierto de Chihuahua (México), donde se comparó el traslape de dietas entre el ganado y el conejo, ambas especies consumieron el mismo porcentaje de herbáceas (31\%) mientras que los conejos consumieron un $47 \%$ de arbustivas y un $22 \%$ de gramíneas contra un $12 \%$ de arbustivas y un $58 \%$ de gramíneas del ganado bovino (Daniel et al. 1993). El índice de Pianka reportado en este trabajo (0.26) es muy similar al publicado por Sangiuliano et al. (2016) cuando compararon el traslape de dieta entre el conejo café (Lepus europaeus) y el corzo (Capreolus capreolus) estos autores reportaron un índice de Pianka de 0.27 cuando estos animales se encontraban en áreas no protegidas y durante el verano debido a la alta disponibilidad y valor nutricional de las especies que consumían. En contraste, los resultados de este trabajo son muy superiores a los publicados por Viviano et al. (2021) quienes con estas mismas especies (conejo café y corzo) reportaron un traslape de 0.15 y explicaron este menor valor debido a la redistribución de los animales en el área de estudio, estos cambios en la distribución hacían que los animales consumieran muchas especies vegetales diferentes. Bajo las condiciones de este trabajo, el traslape de estas dos especies está relacionado con la gran abundancia del Euphorbia schlechtendalii (estomeque) el cual es un componente importante de la dieta de ambas especies. Es importante considerar que el número de lagomorfos estimado es considerablemente más alto que el número de venados cola-blanca y que por esa razón podrían convertirse en un competidor importante de esta especie.
En contraste con los datos de este trabajo, en la región de Michilia (México), el índice de superposición de la dieta entre el venado cola blanca y el ganado fue de $50.51 \%$, lo que sugirió una competencia interespecífica durante la temporada de lluvias, pero debido a la abundancia del forraje se redujeron los conflictos potenciales. Esta reducción en la competencia muestra que, a pesar de presentarse altos niveles de utilización, la vegetación puede mantener una composición más estable cuando es utilizada por dos herbívoros con diferentes preferencias de forraje que cuando es utilizada por un solo (Gallina 1993). Otros estudios muestran que, aunque es más importante económicamente la producción bovina, ésta es compatible con el mantenimiento del venado colablanca siempre que la carga animal se encuentre entre 0.12 y $0.17 \mathrm{UA} \mathrm{ha}^{-1}$ (Hines et al. 2021). Bajo las condiciones de este trabajo, se estimó un coeficiente de agostadero de 33.3 has por animal, lo que representa una carga animal de $0.03 \mathrm{UA} \mathrm{ha}^{-1}$, lo cual se encuentra muy por abajo de los parámetros mencionados anteriormente y justifica el uso económico de ambas especies.

En otro estudio, se comparó el traslape de dieta entre el venado bura (O. hemionus) y los burros salvajes ( $E$. asinus) en el desierto de Sonora, con un índice de superposición de 0.60 . La dieta del venado bura mostró altas proporciones de especies leñosas (76 - 85\%) en todas las estaciones y bajas proporciones tanto de gramíneas ( 1 - $2 \%$ ) como de especies vegetales de hoja ancha (4-8\%), mientras que la dieta del burro salvaje contenía más gramíneas (65 $72 \%$ ) y un porcentaje menor (12 - 16\%) de especies leñosas e intermedio (13 - 20\%) de especies vegetales de hoja ancha (Daniel et al. 1993). Bajo las condiciones de este trabajo, el traslape entre la dieta de los venados cola-blanca y los equinos de la UMA fue de solo 0.04 y está relacionado con el hecho de que la dieta de esta última especie está basada principalmente en el consumo de una herbácea (hierba del golpe) la cual es poco consumida por el venado. 


\section{CONCLUSIONES}

La riqueza de la vegetación en el área sugiere que el manejo de la UMA es adecuado y garantiza el crecimiento de la población de venado cola blanca. El traslape entre la dieta del venado cola blanca, los bovinos y los equinos es poco relevante, sin embargo, el traslape entre venado cola blanca y lagomorfos es del $26 \%$ y considerando el número estimado de individuos en la UMA puede ser un elemento de competencia importante.

\section{LITERATURA CITADA}

Aarssen LW, Laird RA, Pither J (2003) Is the productivity of vegetation plots higher or lower when there are more species? Variable predictions from interaction of 'the'sampling effect' and 'competitive dominance effect' on the habitat templet. Oikos 102: 427-432.

Austin MP (1999) The Potential Contribution of Vegetation Ecology to Biodiversity Research. Ecography 22: 465-484.

Buckland ST, Magurran AE, Green RE, Fewster RM (2005) Monitoring Change in Biodiversity through Composite Indices. Philosophical Transactions Biological Sciences 360: 243-254.

Bullock J (1999) Plants In: Sutherland WJ (ed) Ecological census techniques. A Handbook. Cambridge University Press. Cambridge, UK. pp: 186-213

Burnett M, Hobbs I, Ripple A (2020) Seeing the forest for the deer: Plant abundance and diversity at the Gault nature reserve during a spike in white-tailed deer. McGill Science Undergraduate Research Journal 15: 12-17.

Casas A, Rangel-Landa S, Torres-García I, Peréz-Negrón E, Solis-Rojas L, Parra F, Delgado A, Blancas- Vázquez J, Farfan B, Moreno Calles Al (2008) In situ management and conservation of plant resources in the Tehuacán-Cuicatlán Valley, Mexico: An ethnobotanical and ecological perspective In: De Albuquerque UP, Alves-Ramos M (eds) Current Topics in Ethnobotany. Research Signpost, Kerala India. pp: 1-25.

Campos-Salas N, Casas A, Casas A, Moreno-Calles Al, Vallejo M (2016) Plant Management in Agroforestry Systems of Rosetophyllous Forests in the Tehuacán Valley, Mexico. Economic Botany 70: 254-261.

CONABIO (2011) La Biodiversidad en Puebla: Estudio de Estado. México. Comisión Nacional para el Conocimiento y Uso de la Biodiversidad, Gobierno del Estado de Puebla, Benemérita Universidad Autónoma de Puebla. México. 440p.

Clark A, DelCurto T, Vavra M, Dick BL (2013) Stocking Rate and Fuels Reduction Effects on Beef Cattle Diet Composition and Quality. Rangeland Ecology \& Management 66: 714-720.

Crawford JC, Nielsen CK, Schauber EM (2018) Survival and habitat use of sympatric lagomorphs in bottomland hardwood forests. Canadian Journal of Zoology 96: 713-722.

Daniel A, Holechek JL, Valdez R, Tembo A, Saiwana L, Rusco M, Cardenas M (1993) Range condition influences on Chihuahuan Desert cattle and jackrabbit diets. Journal of Range Management 46: 296-301.

Elzinga CL, Salzer DW, Willoughby JW, Gibbs JP (2001) Monitoring plant and animal populations. Blackwell Science Inc. Massachusetts, USA. 371p.

Faas CJ, Weckerly FW (2010) Habitat Interference by Axis deer on White-Tailed deer. Journal of Wildlife Management 74: 698-706.

Foster SD, Hosack, GR, Monk J, Lawrence E, Barrett NS, Williams A, Przeslawski R (2020) Spatially balanced designs for transect-based surveys. Methods in Ecology and Evolution 11: 95-105. 
Gallina S (1993) White-Tailed deer and cattle diets at La Michilia, Durango, México. Journal of Range Management 46: 487-492.

Guízar-Nolazco E, Granados-Sánchez D, Castañeda-Mendoza A (2010) Flora y vegetación en la porción sur de la mixteca poblana. Revista Chapingo Serie Ciencias Forestales y del Ambiente 16: 95-118.

Hines SL, Fulbright TE, Ortega-S AJ, Webb SL, Hewitt DG, Boutton TW (2021) Compatibility of Dual Enterprises for Cattle and Deer in North America: A Quantitative Review. Rangeland Ecology \& Management 74: 21-31.

Holechek JL (1982) Sample preparation techniques for microhistological analysis. Journal of Range Management 35: 267-268.

Holechek JL, Baker TT, Boren JC, Galt D (2006) Grazing impacts on rangeland vegetation: what we have learned. Livestock grazing at light-to-moderate intensities can have positive impacts on rangeland vegetation in aridto-semiarid areas. Rangelands 28: 7-13.

Howard Jr VW, Cheap KM, Hier RH, Thompson TG, Dimas JA (1987) Effects of Cabling Pinyon-Juniper on mule deer and lagomorph use. Wildlife Society Bulletin 15: 242-247.

Huerta-Martínez FM, Vázquez-García JA, García-Moya E, López-Mata L, Vaquera-Huerta H (2004) Vegetation Ordination at the Southern Chihuahuan Desert (San Luis Potosi, Mexico). Plant Ecology 174: 79-87.

Hulbert IAR, Andersen R (2001) Food competition between a large ruminant and a small hindgut fermentor: the case of the roe deer and mountain hare. Oecologia 128: 499-508.

INEGI (2009) Prontuario de información geográfica municipal de los Estados Unidos Mexicanos, Chiautla Puebla. Clave Geoestadística 21047. Instituto Nacional de Estadística Geografía. México. 9p.

Johnson MK, Wofford H, Pearson HA (1983) Microhistological Techniques for Food Habits Analyses. United States Department of Agriculture. Research Paper SO-1 99 Southern Forest Experiment Station New Orleans. Louisiana, US. 40p.

Julander O (1955) Determining grazing use by cow-chip counts. Journal of Range Management 8: 182

Kingery JI, Mosley JC, Bordwell KC (1996) Dietary overlap among cattle and cervids in northern Idaho forests. Journal of Range Management 49: 8-15.

Kontsiotis VJ, Bakaloudis DE, Merou T, Xofis P (2015) Trophic ecology of the European wild rabbit Oryctolagus cuniculus on the Mediterranean island of Lemnos, Greece Ecological Research 30: 683-691

Krebs CJ (2006) Mammals. In: Sutherland WJ (ed) Ecological census techniques: A Handbook. Second Edition. Cambridge University Press. Cambridge, UK. pp: 351-369.

Mandujano S (2011) Consideraciones ecológicas para el manejo del venado cola blanca en UMA extensiva en bosques tropicales. En: Sánchez Ó, Zamorano P, Peters E, Moya H (eds) Temas sobre conservación de vertebrados silvestres en México. Secretaría de Medio Ambiente y Recursos Naturales, Instituto Nacional de Ecología. México. pp: 249-275.

Mandujano S (2014) PELLET: An Excel ${ }^{\circledR}$-based procedure for estimating deer population density using the pellet-group counting method. Tropical Conservation Science 7: 308-325.

Marques FFC, Buckland ST, Goffin D, Dixon CE, Borchers DL, Mayle BA, Peace AJ (2001) Estimating deer abundance from line transect surveys of dung: sika deer in southern Scotland. Journal of Applied Ecology 38: $349-363$ 
Marshal JP, Bleich VC, Krausman PR, Reed ML, Neibergs A (2012) Overlap in diet and habitat between the mule deer (Odocoileus hemionus) and feral ass (Equus asinus) in the Sonoran desert. The Southwestern Naturalist 57: 16-25.

Martínez MA, Molina V, González F, Marroquín S, Navar J (1997) Observations of white-tailed deer and cattle diets in México. Journal of Range Management 50: 253- 257.

Martínez-Calderas JM, Palacio-Núñez J, Martínez-Montoya JF, Clemente-Sánchez F, Sánchez-Rojas G, OlmosOropeza G (2016) Abundancia relativa y distribución de Lagomorfos en el centro-norte de México. Agroproductividad 9: 67-72.

Martínez-García JA, Mendoza-Martínez GD, Alcantara-Carbajal JL, Tarango-Arámbula LA, Sánchez-Torres-Esqueda T, Rodríguez-de Lara R, Hernández-García PA (2012) Composición de la dieta y capacidad de carga del hábitat del conejo de los volcanes (Romerolagus diazi) en México. Revista Chapingo Serie Ciencias Forestales y del Ambiente 18: 423-434.

Matteucci SD, Colma A, Pla L (1999) Biodiversidad vegetal en el árido falconiano (Venezuela). Interciencia 24: 300-307.

Mayle BA, Peace AJ, Gill RMA (1999) How many deer? A field guide to estimating deer population size. Field Book. Edinburgh Forestry Commission. UK. 97p.

McCain CM, King SRB, Szewczyk T, Beck J (2018) Small mammal species richness is directly linked to regional productivity, but decoupled from food resources, abundance, or habitat complexity. Journal of Biogeography 45: 2533-2545.

Mutze G, Cooke B, Lethbridge M, Jennings S (2014) A rapid survey method for estimating population density of European rabbits living in native vegetation. The Rangeland Journal 36: 239-247

Otto R, Fernández-Palacios JM, Krüsi BO (2001) Variation in species composition and vegetation structure of succulent scrub on Tenerife in relation to environmental variation. Journal of Vegetation Science 12: 237248.

Plata FX, Ebergeny S, Resendiz JL, Villarreal O, Bárcena R, Viccon JA, Mendoza GD (2009) Palatabilidad y composición química de alimentos consumidos en cautiverio por el venado cola blanca de Yucatán (Odocoileus virginianus yucatanensis). Archivos de Medicina Veterinaria 41: 123-129.

Plata FX, Mendoza GD, Viccon JA, Bárcena R, Sánchez FC, Villarreal OA (2011) Adecuación y análisis de sensibilidad de un modelo para la estimación de la capacidad de carga del hábitat de venado cola blanca. Archivos de Medicina Veterinaria 43: 267-275.

Peña JM, Habib R (1980) La técnica microhistológica; Un método para determinar la composición botánica de la dieta de herbívoros. Instituto Nacional de Investigaciones Pecuarias, Secretaría de Agricultura y Recursos Hidráulicos. Serie Técnico Científica Vol. 1, Nº 6. México. 180p.

Portales-Betancourt GL, Hernández-Laundré L, Laundré JW, Cervantes FA (2016) Reproducción y densidad de la liebre cola-negra (Lepus californicus) en relación con factores ambientales, en la Reserva de la Biosfera Mapimí, Desierto Chihuahuense. Therya 3: 171-183.

Pruszenski JM, Hernández DL (2020) White-tailed Deer Fecal Matter Distribution and Nutrient Contribution in Tallgrass Prairie. American Midland Naturalist 184: 268-273.

Rivero MJ, Grau-Campanario P, Mullan S, Held SDE, Stokes JE, Lee MRF, Cardenas LM (2021) Factors Affecting Site Use Preference of Grazing Cattle Studied from 2000 to 2020 through GPS Tracking: A Review. Sensors 21: 2696. DOI: 10.3390/s21082696. 
Sánchez-Rojas G, Gallina S, Equihua M (2004) Pellet morphometry as tool to distinguish age and sex in the mule deer. Zoo Biology 23: 139-146.

Sangiuliano A, Lovari S, Ferretti F (2016) Dietary partitioning between European roe deer and European brown hare. European Journal of Wildlife Research 62: 527-535.

Villareal GJ (1999) Venado Cola Blanca. Manejo y aprovechamiento cinegético. Unión Ganadera Regional de Nuevo León, México. 263p.

Villarreal Espino-Barros OA, Guevara Viera R, Reséndiz Martínez R, Hernández Zepeda JS, Castillo Correo JC, Tomé Torres FJ (2005) Diversificación productiva en el campo experimental las Margaritas, Puebla, México. Archivos de Zootecnia 54: 197-203.

Villarreal Espino-Barros OA (2006) El venado cola blanca en la Mixteca Poblana. Conceptos y métodos para su conservación y manejo. Fundación Produce Puebla. Benemérita Universidad Autónoma de Puebla, Mazamiztli AC. México. 191p.

Villarreal OA, Plata FX, Camacho JC, Hernández JE, Franco FJ, Aguilar B, Mendoza GD (2011) El venado cola blanca en la Mixteca Poblana. Therya 2: 103-110.

Viviano A, Mori E, Fattorini N, Mazza G, Lazzeri L, Panichi A, Strianese L, Mohamed WF (2021) Spatiotemporal overlap between the European Brown Hare and its potential predators and competitors. Animals 11: 562. DOI: 10.3390/ani11020562.

Williams K, Parer I, Coman B, Burley J, Braysher M (1995) Managing vertebrate pests: Rabbits. Australian Government Publishing Service. Canberra, Australia. 284p.

Zabek MA, Berman DM, Blomberg S, Wright J (2016) Estimating distribution and abundance of feral horses (Equus caballus) in a coniferous plantation in Australia, using line-transect surveys of dung. Wildlife Research 43: 604-614. 